\title{
A Globally Stabilising Controller under Saturated Input for Linear Planar Systems with One Unstable Pole
}

\author{
J-Y. Favez, Ph. Mullhaupt, B. Srinivasan, D. Bonvin \\ Laboratoire d'Automatique, Ecole Polytechnique Fédérale de Lausanne, \\ $\mathrm{CH}-1015$ Lausanne, Switzerland \\ email: jean-yves.favez@epfl.ch; fax: +41216932574
}

\begin{abstract}
Controllers for planar systems with one stable and one unstable pole under saturated input are considered. The requirements are: (i) global stability, i.e. the region of attraction is the null controllable region, (ii) possibility of enforcing any desired performance around the origin, and (iii) no chattering of the control signal in the presence of noise. A simple continuous nonlinear state feedback controller is proposed that satisfies all these requirements. The performance of the proposed controller is compared in simulation with that of classical controllers such as linear state feedback and time optimal controllers.
\end{abstract}

Keywords: Region of Attraction, Saturated Input, Continuous Nonlinear Control, Unstable Planar Systems.

\section{INTRODUCTION}

Linear systems with bounded inputs have been widely studied in the literature [9], [6], [4]. This type of studies is important since, in most practical situations, the range of inputs is in fact limited.

In this paper, the control of single-input linear planar systems (systems with 2 states) with saturated linear and nonlinear feedback will be considered. Especially, the emphasis will be on systems with one stable and one unstable pole. The three main requirements addressed here are as follows:

1) Global stability: Two important concepts pertaining to these systems have to be distinguished. First is the null controllable region $\mathcal{C}$, i.e. the region in state space where there exists an open-loop input that can steer the system to the origin [1], [4], [5], [8]. The second is the region of attraction $\mathcal{A}$ with a given controller, i.e. the region in state space from which the closedloop system asymptotically reaches the origin [1], [4]. A controller is globally stabilising when $\mathcal{A}=\mathcal{C}$.

For semi-stable planar systems (both poles have nonpositive real parts), $\mathcal{C}=\mathbb{R}^{2}$ and $\mathcal{A}=\mathcal{C}=\mathbb{R}^{2}$ for any linear state feedback which makes the origin globally asymptotically stable in the absence of saturation [9]. However, for systems with one stable and one unstable pole, $\mathcal{C} \subset \mathbb{R}^{2}$ and $\mathcal{A}=\mathcal{C}$ can be either achieved with an optimal or near optimal variable structure control (VSC) [7], [8] or using a linear state feedback controller where only the unstable state is fed back [1], [8]. For anti-stable systems (both poles have positive real parts), $\mathcal{C} \subset \mathbb{R}^{2}$ and the only way to obtain $\mathcal{A}=\mathcal{C}$ is through optimal or near optimal switching [4], [5].
2) Local performance - Enforcing the desired performance locally around the origin: For systems with one stable and one unstable pole, a simple way of obtaining global stability is to feed back only the unstable state. However, such a controller will have poor local performance due to absence of feedback on the stable mode. The proposed controller should guarantee, at least locally, the desired temporal performance.

3) Absence of chattering in the control signal in the presence of noise: From the point of view of the above two requirements, the optimal or near optimal switching strategies are the methods of choice. The controllers have the maximum region of attraction and provide excellent time performance, not only locally, but even globally. However, the main problem is that the control signal is chattering in the presence of noise. Absence of chattering is a requirement for the sought controller.

In what follows various standard controllers proposed in the literature are analysed from the perspective of control of linear planar systems with one stable and one unstable pole.

i) Standard linear state feedback controller where both states are fed back [3]: Requirement 1 is not satisfied.

ii) Linear state feedback controllers where only the unstable state is fed back [1], [8]: Requirement 2 is not satisfied since the stable state is left to follow its own dynamics.

iii) Optimal or near-time optimal VSC controller [7], [8]: Requirement 3 is not verified in presence of noise and disturbances.

iv) Controller switching from controller (ii) to controller (i) [4], [5]: When the state reaches an invariant nonsaturated region, the controller switches from type (ii) to (i). The problem is that the invariant region could be very small.

The properties of these controllers are summarised in Table I. The controllers available in the literature cannot satisfy one or more of the requirements stated above. Thus, a controller is proposed that meets all the aforementioned requirements. The idea is to have a controller of type (iv) with a continuous switching from controller type (ii) to controller type (i). This way, the problem of chattering is avoided and the region of attraction is the whole null 
controllable region $\mathcal{A}=\mathcal{C}$.

\begin{tabular}{|l||c|c|c|}
\hline & $\begin{array}{c}\text { Global } \\
\text { stability }\end{array}$ & $\begin{array}{c}\text { Local } \\
\text { performance }\end{array}$ & $\begin{array}{c}\text { No } \\
\text { chattering }\end{array}$ \\
\hline \hline Standard linear & - & $\sqrt{ }$ & $\sqrt{ }$ \\
\hline Feedback unstable state & $\sqrt{ }$ & - & $\sqrt{ }$ \\
\hline Optimal controller & $\sqrt{ }$ & $\sqrt{ }$ & - \\
\hline Switching controller & $\sqrt{ }$ & - & $\sqrt{ }$ \\
\hline
\end{tabular}

TABLE I

PROPERTIES OF THE STATE OF THE ART CONTROLLERS.

The paper is organised as follows. In Section II, definitions and terms used in this paper are introduced. Section III provides the main idea behind the structure of the proposed continuous nonlinear controller. In Section IV, the global stabilisation in the null controllable region is discussed. Section V compares the proposed controller against the standard approaches. Conclusions are drawn in Section VI.

\section{Preliminaries}

\section{A. Linear planar system with input saturation}

Consider a single-input second-order linear system with a stable and an unstable pole. Upon state transformation, the system can be written as:

$$
\dot{x}=A x+b u=\left[\begin{array}{cc}
\lambda_{1} & 0 \\
0 & \lambda_{2}
\end{array}\right] x+\left[\begin{array}{c}
\lambda_{1} \\
\lambda_{2}
\end{array}\right] u
$$

where, $x \in \mathbb{R}^{2}$ is the state vector, $u$ the input, $A$ and $b$ appropriate matrices, and $\lambda_{1}, \lambda_{2}$ the eigenvalues of the open-loop system. Assume that $\lambda_{1}>0$ and $\lambda_{2}<0$. The symmetric saturation function with unity saturation level will be used:

$$
\operatorname{sat}(s)= \begin{cases}-1 & \text { if } \quad s<-1 \\ s & \text { if } \quad-1 \leq s \leq 1 \\ 1 & \text { if } \quad s>1\end{cases}
$$

With saturated state feedback, the closed-loop system is

$$
\dot{x}=A x+b \operatorname{sat}(\tilde{u}(x)),
$$

where $\tilde{u}(x): \mathbb{R}^{2} \rightarrow \mathbb{R}$ is the linear or nonlinear control law in function of the states.

\section{B. Equilibrium points and region of attraction}

Assume that the feedback $\tilde{u}(x)$ is a global stabiliser for (1). Then, system (3) has three equilibrium points. This conclusion, which regards an open-loop system with one stable and one unstable pole, does not apply to all other open-loop pole configurations (both poles stable or unstable) where the origin is the unique equilibrium point [1].

Theorem 1 [1] Let the feedback $\tilde{u}$ be a global stabiliser for (1) at the origin. Then, the closed-loop system (3) has three equilibrium points: $x_{e+}=A^{-1} b=\left[\begin{array}{ll}1 & 1\end{array}\right]^{T}, x_{e-}=$ $-A^{-1} b=\left[\begin{array}{ll}-1 & -1\end{array}\right]^{T}$, and $x_{e 0}=0$. Of these, $x_{e 0}$ is stable, while the other two are saddle points.
Definition 1 Let $\Phi\left(t, x_{0}\right)$ denote the state of (3) at time $t$, starting with the initial condition $x_{0}$ at $t=0$. The region of attraction of the stable equilibrium point $x_{e 0}=0$ is defined as:

$$
\mathcal{A}=\left\{x: \lim _{t \rightarrow \infty} \Phi(t, x)=0\right\} .
$$

The boundary of $\mathcal{A}$ is denoted by $\partial \mathcal{A}$.

\section{Manifolds}

Define the following hyperplanes and manifolds (refer to Figure 1 for illustration):

- $\partial \mathcal{L}_{0}=\{x: \tilde{u}=0\}$

- $\partial \mathcal{L}_{+}=\{x: \tilde{u}=1\}, \partial \mathcal{L}_{-}=\{x: \tilde{u}=-1\}$

- $\partial \mathcal{C}_{+}=\left\{x: x_{1}=1\right\}, \partial \mathcal{C}_{-}=\left\{x: x_{1}=-1\right\}$

- $\partial \mathcal{S}_{+}=\left\{x: \lim _{t \rightarrow \infty} \Phi(t, x)=x_{e+}\right\}$

- $\partial \mathcal{S}_{-}=\left\{x: \lim _{t \rightarrow \infty} \Phi(t, x)=x_{e-}\right\}$

The hyperplanes $\partial \mathcal{L}_{+}$and $\partial \mathcal{L}_{-}$are the boundaries of the region $\mathcal{L}$ where the control is not saturated and $\partial \mathcal{L}_{0}$ is the hyperplane of zero control. The hyperplanes $\partial \mathcal{C}_{+}$and $\partial \mathcal{C}_{-}$ are the boundaries of null controllable region $\mathcal{C}$ [1], [8]. $\partial \mathcal{S}$ denote the stable manifolds of the saddle points. All manifolds have two branches, one on either side of the saddle points.

\section{Continuous Nonlinear State Feedback CONTROLLER}

Consider the controller

$$
\tilde{u}(x)=f_{1} x_{1}+k(x) f_{2} x_{2} \quad u=\operatorname{sat}(\tilde{u}),
$$

where $f=\left[\begin{array}{ll}f_{1} & f_{2}\end{array}\right] \in \mathbb{R}^{2}$ and $k(x): \mathbb{R}^{2} \rightarrow \mathbb{R}$. Assume that $f$ has been chosen to get the desired performance (Requirement 2) of the closed-loop system near the origin. If we set $k(x)=1$, then (5) is a linear state feedback controller. If $k(x)=0$, then the stable state is not fed back leading to $\mathcal{A}=\mathcal{C}$ [1]. Here, a continuous switching is introduced by choosing:

$$
k(x)=1-\left|x_{1}\right|,
$$

where $0<k(x) \leq 1$ since within the null controllable region $\left|x_{1}\right|<1$.

The idea behind this nonlinear controller is as follows. If $x_{1} \approx 0$, then $k(x) \approx 1$ which implies that the controller is approximately the linear state feedback $\tilde{u} \approx f_{1} x_{1}+f_{2} x_{2}$. In this case, the controller concentrates on local performance (Requirement 2). On the contrary, if the unstable state approaches the boundary of the null controllable region $\mathcal{C}$, $x_{1} \approx \pm 1$ and $k(x) \approx 0$. This implies that the controller is approximately the linear state feedback $\tilde{u} \approx f_{1} x_{1}$, where it focuses on the stabilisation of the unstable state and global stability (Requirement 1). Since the controller (5)-(6) is a continuous one, chattering is avoided and Requirement 3 is also fulfilled.

Since $f$ stabilises the system locally, it satisfies the Hurwitz stability conditions:

1) $\lambda_{1}\left(1+f_{1}\right)+\lambda_{2}\left(1+f_{2}\right)<0$

2) $\lambda_{1} \lambda_{2}\left(1+f_{1}+f_{2}\right)>0,\left(1+f_{1}+f_{2}\right)<0$ 
Also the condition that the system is stable at $k(x)=0$ implies $\left(1+f_{1}\right)<0$.

To picture the regions where the control is saturated and where it is not, consider the function $g_{\tilde{u}}:(-1,1) \rightarrow \mathbb{R}$ that describes $x_{2}$ as a function of $x_{1}$ for a given $\tilde{u} \in[-1,1]$ :

$$
x_{2}=g_{\tilde{u}}\left(x_{1}\right)=\frac{\tilde{u}-f_{1} x_{1}}{f_{2}\left(1-\left|x_{1}\right|\right)} .
$$

With this function, it is possible to express $\partial \mathcal{L}_{0}, \partial \mathcal{L}_{+}$and $\partial \mathcal{L}_{-}$by setting $\tilde{u}=0, \tilde{u}=1$ and $\tilde{u}=-1$, respectively:

$$
\begin{array}{r}
\partial \mathcal{L}_{0}=\left\{x \in(-1,1) \times \mathbb{R}: x_{2}=\frac{-f_{1} x_{1}}{f_{2}\left(1-\left|x_{1}\right|\right)}\right\} \\
\partial \mathcal{L}_{+}=\left\{x \in(-1,1) \times \mathbb{R}: x_{2}=\frac{1-f_{1} x_{1}}{f_{2}\left(1-\left|x_{1}\right|\right)}\right\} \\
\partial \mathcal{L}_{-}=\left\{x \in(-1,1) \times \mathbb{R}: x_{2}=\frac{-1-f_{1} x_{1}}{f_{2}\left(1-\left|x_{1}\right|\right)}\right\}
\end{array}
$$

Figure 1 illustrates $\partial \mathcal{L}_{0}, \partial \mathcal{L}_{+}, \partial \mathcal{L}_{-}$, the boundary of the null controllable region $\partial \mathcal{C}_{+}$and $\partial \mathcal{C}_{-}$, and the equilibrium saddle nodes $x_{e+}$ and $x_{e-}$.

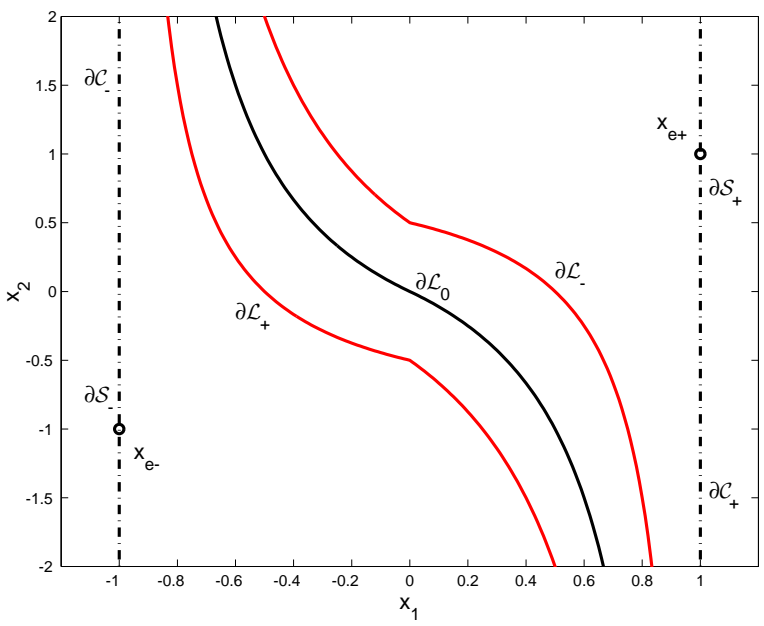

Fig. 1. Illustration of $\partial \mathcal{L}_{0}, \partial \mathcal{L}_{+}, \partial \mathcal{L}_{-}$, the boundary of the null controllable region $\partial \mathcal{C}_{+}$and $\partial \mathcal{C}_{-}$, the stable manifolds $\partial \mathcal{S}_{+}$and $\partial \mathcal{S}_{-}$, and the equilibrium saddle nodes $x_{e}$ and $x_{e-}$.

\section{Analysis of the Proposed CONTROller}

Consider the closed-loop system with (3), (5) and (6) :

$$
\dot{x}=A x+b \operatorname{sat}\left(f_{1} x_{1}+f_{2} x_{2}\left(1-\left|x_{1}\right|\right)\right)
$$

It was shown in [3] that the stable manifolds are the boundaries of the region of attraction $\mathcal{A}$. In what follows, it will be shown that, with controller (5) the stable manifolds are in fact the boundaries of the null controllable region $\mathcal{C}$.

Proposition 1 Consider system (8). If $\left(1+f_{1}\right)<0$, then

$$
\partial \mathcal{S}_{+}=\partial \mathcal{C}_{+} \text {and } \partial \mathcal{S}_{-}=\partial \mathcal{C}_{-}
$$

Proof: Consider the input (5) along the manifold $\partial \mathcal{C}_{+}$: $u=\operatorname{sat}\left(f_{1}\right)=-1$, since $f_{1}<-1$. Then, (8) along $\partial \mathcal{C}_{+}$ reads:

$$
\begin{gathered}
\dot{x}_{1}=\lambda_{1}\left(x_{1}-1\right)=0 \\
\dot{x}_{2}=\lambda_{2}\left(x_{2}-1\right)
\end{gathered}
$$

Thus, $x_{1}$ stays at 1 , and since $\lambda_{2}<0, x_{2}$ converges to 1 as well. Thus, $\partial \mathcal{C}_{+}$forms the set of all points that converge to the equilibrium point $x_{e+}$. So, $\partial \mathcal{S}_{+}=\partial \mathcal{C}_{+}$. A similar proof can be written for $\partial \mathcal{S}_{-}=\partial \mathcal{C}_{-}$.

Lemma 1 Let $X=(-1,1) \times[-1,1]$ and $D=[-1+\epsilon, 1-$ $\epsilon] \times[-1,1]$, with $\epsilon>0$ chosen such that $|u(x)|=1$ for all $x \in(X \backslash D)$. Then, all trajectories starting in $\mathcal{C}$ will enter the compact invariant set $D$.

Proof: Consider the subset $X_{+}=(-1,1) \times(1, \infty]$. Within this set,

$$
\dot{x}_{2}=\lambda_{2}\left(x_{2}+u\right)<0
$$

since $\lambda_{2}<0, x_{2}>1$, and $|u| \leq 1$ due to saturation. So, all trajectories starting in $X_{+}$will leave $X_{+}$. A similar argument can be provided for $X_{+}=(-1,1) \times[-\infty,-1)$. So, since $\mathcal{C}$ is invariant, all trajectories starting in $\mathcal{C}$ will reach $X=\mathcal{C} \backslash\left(X_{+} \cup X_{-}\right)=(-1,1) \times[-1,1]$.

Consider the subset $Y_{-}=(-1,-1+\epsilon) \times[-1,1]$, with $\epsilon>0$ chosen such that $u(x)=1$ for all $x \in Y_{-}$. So, within this set

$$
\dot{x}_{1}=\lambda_{1}\left(x_{1}+u\right)>0
$$

since $\lambda_{1}>0, x_{1}>-1$, and $u=1$. Thus, eliminating $Y_{-}$and its counterpart $Y_{+}=(1,1-\epsilon) \times[-1,1]$ gives the compact invariant set $D=X \backslash\left(Y_{+} \cup Y_{-}\right)$. So, every trajectory starting in $X$ reaches $D$.

The proof is complete since every trajectory starting in $\mathcal{C}$ reaches $X$ and every trajectory starting in $X$ reaches $D$. Thus, $D$ is invariant.

Lemma 2 If $\lambda_{1}+\lambda_{2}>0$ or $\left(f_{2}-f_{1}\right) \leq 0$, then the nonsaturated region $\mathcal{L}$ is an invariant set for (8).

Proof: The lemma is proved by showing that the vector field of $(8)$ points into the non-saturated region $\mathcal{L}$ for every point along the manifolds $\partial \mathcal{L}_{+}$and $\partial \mathcal{L}_{-}$. Since $\partial \mathcal{L}_{+}$and $\partial \mathcal{L}_{-}$are symmetric it suffices to prove it only for one manifold $\left(\partial \mathcal{L}_{+}, \tilde{u}=1\right)$.

The slope of the tangent of $\partial \mathcal{L}_{+}, s_{1}$, is given by:

$$
s_{1}=\frac{d x_{2}}{d x_{1}}=\frac{d g_{\tilde{u}}\left(x_{1}\right)}{d x_{1}}=\frac{-f_{1}+\operatorname{sgn}\left(x_{1}\right)}{f_{2}\left(1-\left|x_{1}\right|\right)^{2}},
$$

Note that $s_{1}<0$ for $f_{2}<0$ and $s_{1}>0$ for $f_{2}>0$.

Noting that $x_{2}=g_{\tilde{u}}\left(x_{1}\right)$ along $\partial \mathcal{L}_{+}$, the slope of the vector field $s_{2}$ is given by:

$$
s_{2}=\frac{\dot{x}_{2}}{\dot{x}_{1}}=\frac{\lambda_{2}\left(x_{2}+1\right)}{\lambda_{1}\left(x_{1}+1\right)}=\frac{\lambda_{2}}{\lambda_{1}} \frac{1-f_{1} x_{1}+f_{2}\left(1-\left|x_{1}\right|\right)}{f_{2}\left(1-\left|x_{1}\right|\right)\left(1+x_{1}\right)}
$$


The vector field points into non-saturated region $\mathcal{L}$ if $s_{2}>$ $s_{1}$ for $s_{1}<0\left(f_{2}<0\right)$ and $s_{2}<s_{1}$ for $s_{1}>0\left(f_{2}>0\right)$. Since both $s_{1}$ and $s_{2}$ have $f_{2}$ in the denominator, the two cases with $f_{2}>0$ and $f_{2}<0$ can be unified to give the following condition:

$$
\frac{\lambda_{2}}{\lambda_{1}} \frac{1-f_{1} x_{1}+f_{2}\left(1-\left|x_{1}\right|\right)}{\left(1-\left|x_{1}\right|\right)\left(1+x_{1}\right)}<\frac{-f_{1}+\operatorname{sgn}\left(x_{1}\right)}{\left(1-\left|x_{1}\right|\right)^{2}} .
$$

Since $\left(1+x_{1}\right) \geq\left(1-\left|x_{1}\right|\right)>0$ and $\left(-f_{1}+\operatorname{sgn}\left(x_{1}\right)\right)>$ $\left(-f_{1}-1\right)$, the condition can be rearranged to give:

$$
\lambda_{1}\left(1+f_{1}\right)+\lambda_{2}\left(1+f_{2}\left(1-\left|x_{1}\right|\right)\right)-\lambda_{2} f_{1} x_{1}<0
$$

So, the lemma is proved if it can be shown that (13) is verified.

Case 1: $x_{1} \geq 0$

From the Hurwitz stability condition, $\lambda_{1}\left(1+f_{1}\right)+\lambda_{2}(1+$ $\left.f_{2}\left(1-\left|x_{1}\right|\right)\right)<0$. Since $\lambda_{2}<0$ and $f_{1}<0$ the last term of (13), $-\lambda_{2} f_{1} x_{1} \leq 0$. So, the inequality (13) holds.

Case 2: $x_{1}<0$

Substituting $\left|x_{1}\right|=-x_{1}$ in (13) reads:

$\phi\left(x_{1}\right)=\lambda_{1}\left(1+f_{1}\right)+\lambda_{2}\left(1+f_{2}\right)+\lambda_{2} x_{1}\left(f_{2}-f_{1}\right)<0$

Due to linearity, the maximum of $\phi\left(x_{1}\right) \forall-1 \leq x_{1} \leq 0$ occurs either at $x_{1}=-1$ or $x_{1}=0$ depending on the sign of $\left(f_{2}-f_{1}\right)$. If $\left(f_{2}-f_{1}\right) \leq 0$, the maximum is at $x_{1}=0$. Thus, it is required to prove $\lambda_{1}\left(1+f_{1}\right)+\lambda_{2}\left(1+f_{2}\right)<0$, which is verified since it is the Hurwitz stability condition. If $\left(f_{2}-f_{1}\right)>0$, the maximum is at $x_{1}=-1$, and the inequality (14) becomes:

$$
\left(\lambda_{1}+\lambda_{2}\right)\left(1+f_{1}\right)<0 .
$$

Due to the Hurwitz stability condition, $\left(1+f_{1}\right)<0$, and due to the hypothesis, $\left(\lambda_{1}+\lambda_{2}\right)>0$, this condition is verified.

The next theorem is the main result where the global asymptotic stability in $\mathcal{C}$ is guaranteed under the condition

$$
\lambda_{1}\left(2+f_{1}\right)+\lambda_{2}\left(1+f_{2}\right)<0 .
$$

Since $\lambda_{1}>0$, this is a slightly more restrictive condition than the Hurwitz condition $\lambda_{1}\left(1+f_{1}\right)+\lambda_{2}\left(1+f_{2}\right)<0$.

\section{Theorem 2 If $f$ satisfies the conditions}

$$
\left(1+f_{1}+f_{2}\right)<0, \quad \lambda_{1}\left(2+f_{1}\right)+\lambda_{2}\left(1+f_{2}\right)<0,
$$

then, the closed loop system

$$
\dot{x}=h(x)=A x+b \operatorname{sat}\left(f_{1} x_{1}+f_{2} x_{2}\left(1-\left|x_{1}\right|\right)\right)
$$

is asymptotically stable for all initial conditions in $\mathcal{C}$.

Proof: First it is shown, using an extension of the Bendixson's theorem [2], that no limit cycle exists. The extension of the Bendixson's theorem claims that if on a compact invariant set $F$, the divergence $\nabla h$ defined by:

$$
\nabla h(x)=\frac{\partial \dot{x}_{1}}{\partial x_{1}}+\frac{\partial \dot{x}_{2}}{\partial x_{2}}
$$

exists and has the same sign almost everywhere in $F$, then $F$ contains no closed trajectories.

For the non-saturated region without the boundaries $(\mathcal{L} \backslash$ $\left.\left(\partial \mathcal{L}_{+} \cup \partial \mathcal{L}_{-}\right)\right)$, using the condition (16), it can be seen that

$$
\begin{aligned}
\nabla h(x)= & \lambda_{1}\left(1+f_{1}\right)+\lambda_{2}\left(1+f_{2}\left(1-\left|x_{1}\right|\right)\right) \\
& -\lambda_{1} f_{2} x_{2} \operatorname{sgn}\left(x_{1}\right)<0
\end{aligned}
$$

For the compact saturated region $(D \backslash \mathcal{L})$

$$
\nabla h(x)=\lambda_{1}+\lambda_{2}
$$

Case 1: $\lambda_{1}+\lambda_{2}>0$

In this case, the non-saturated region $\mathcal{L}$ is invariant (Lemma 2). Since $\nabla h<0$ in the invariant region and there are no limit cycles.

Case 2: $\lambda_{1}+\lambda_{2} \leq 0$

In this case, $\nabla h$ is non-positive in both the saturated and non-saturated regions, and so there are no limit cycles either.

The proof of the theorem is now based on the application of the Poincare-Bendixson's theorem within the compact invariant set $D$. So, every trajectory of (17) starting at $x_{0} \in$ $\mathcal{C}$ will either i) go to the equilibrium point at the origin, ii) tend to a limit cycle or iii) be a limit cycle itself. In the first part of the proof, it has been shown that there are no limit cycles. So, all trajectories in $\mathcal{C}$ converge asymptotically to the origin. Thus, the region of attraction is in fact $\mathcal{A}=\mathcal{C}$.

\section{Comparison of Controllers via Simulations}

System (1) is considered with the parameters $\lambda_{1}=1$ and $\lambda_{2}=-0.5$, i.e.

$$
\begin{aligned}
& \dot{x}_{1}=x_{1}+u \\
& \dot{x}_{2}=-0.5\left(x_{2}+u\right)
\end{aligned}
$$

The following 4 controllers are compared:

1) C1: Linear saturated controller: $u=\operatorname{sat}\left(f_{1} x_{1}+f_{2} x_{2}\right), f_{1}=-6$ and $f_{2}=-3$

2) C2: Linear saturated controller with only $x_{1}$ fed back: $u=\operatorname{sat}\left(f_{1} x_{1}\right), f_{1}=-3$

3) C3: Time optimal controller [7]: Switching between $u=-1$ and $u=1$

4) C4: Continuous nonlinear controller: $u=\operatorname{sat}\left(f_{1} x_{1}+f_{2} x_{2}\left(1-\left|x_{1}\right|\right)\right), f_{1}=-6$ and $f_{2}=-3$

The controllers $\mathrm{C} 1$ and $\mathrm{C} 4$ are tuned such that both closedloop poles of the linear system are place at -2 . As far as C2 is concerned, only the pole corresponding to the unstable mode can be influenced by the controller and this pole is placed at -2 . The switching control law for C3 is given by 
the following set of equations:

$$
\begin{gathered}
u_{s}=\operatorname{sgn}\left(\left[\begin{array}{ll}
-1 & 1
\end{array}\right] x\right) \\
\tilde{x}=\left[\begin{array}{cc}
\left(\frac{\tilde{x}_{0_{1}}}{\tilde{x}_{0_{2}}}\right)^{\frac{\lambda_{1}}{\lambda_{2}-\lambda 1}} & 0 \\
0 & \left(\frac{\tilde{x}_{0_{1}}}{\tilde{x}_{0_{2}}}\right)^{\frac{\lambda_{2}}{\lambda_{2}-\lambda 1}}
\end{array}\right] \tilde{x}_{0} \\
\delta=\operatorname{sgn}\left(\left\|x_{e+}\right\|-\|\tilde{x}\|\right) \\
u= \begin{cases}u_{s} & \text { if } \delta=0 \\
u_{s} \delta & \text { if } \delta \neq 0\end{cases}
\end{gathered}
$$

The three properties mentioned in the introduction, i.e. (i) global stability, (ii) local performance, and (iii) chattering behavior are analysed for these four controllers.

\section{A. Global stability}

In Figure 2, the evolution of the closed-loop trajectories for the initial condition $x_{0}=\left[\begin{array}{ll}-0.8 & 2.8\end{array}\right]^{T}$ is illustrated. This initial condition is not in the region of attraction $\mathcal{A}$ of $\mathrm{C} 1$ and therefore the corresponding trajectory leaves the null controllable region $\mathcal{C}$ and escapes to infinity. However, all the other controllers are globally stable with $\mathcal{A}=\mathcal{C}$ and thus the corresponding trajectories converge to the origin.

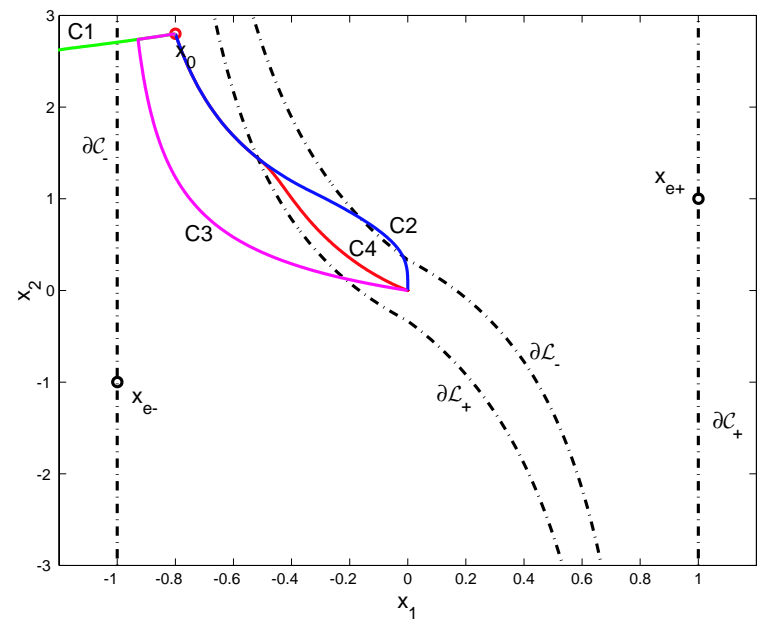

Fig. 2. Phase diagram. The lines $\mathcal{L}_{+}$and $\mathcal{L}_{-}$are the borders of the non-saturated region of controller $\mathrm{C} 4$.

\section{B. Local performance}

In Figure 3, the evolution of the trajectories of all controllers for the initial condition $x_{0}=\left[\begin{array}{cc}0.7 & 2.8\end{array}\right]^{T} \in \mathcal{C}$ is shown. It is seen that the trajectory of controller $\mathrm{C} 2$ first goes towards the $x_{2}$ axis since only the unstable state $x_{1}$ is fed back. Then, convergence to the origin is ensured via the open-loop dynamics of the stable state.

The time evolution of the two states is shown in Figure 4. For controller $\mathrm{C} 2$, the unstable state is damped quickly.
However, the damping of the stable state takes a long time. The best time performance for both states is given by the time-optimal controller $\mathrm{C} 3$. The controller $\mathrm{C} 4$ needs more time to damp the states but does better than $\mathrm{C} 1$. For initial conditions that are located nearer the origin, the performance of $\mathrm{C} 4$ and $\mathrm{C} 1$ is similar.

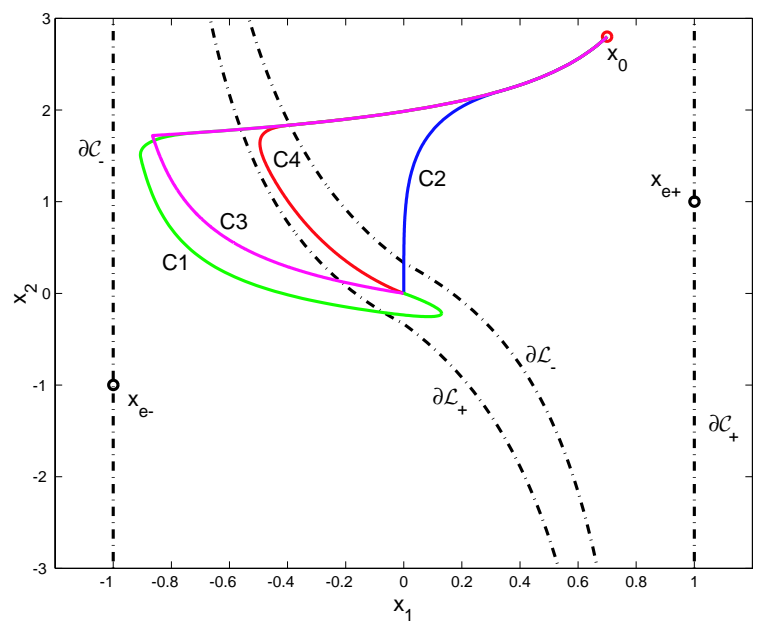

Fig. 3. Phase diagram. The lines $\mathcal{L}_{+}$and $\mathcal{L}_{-}$are the borders of the non-saturated region of controller $\mathrm{C} 4$.
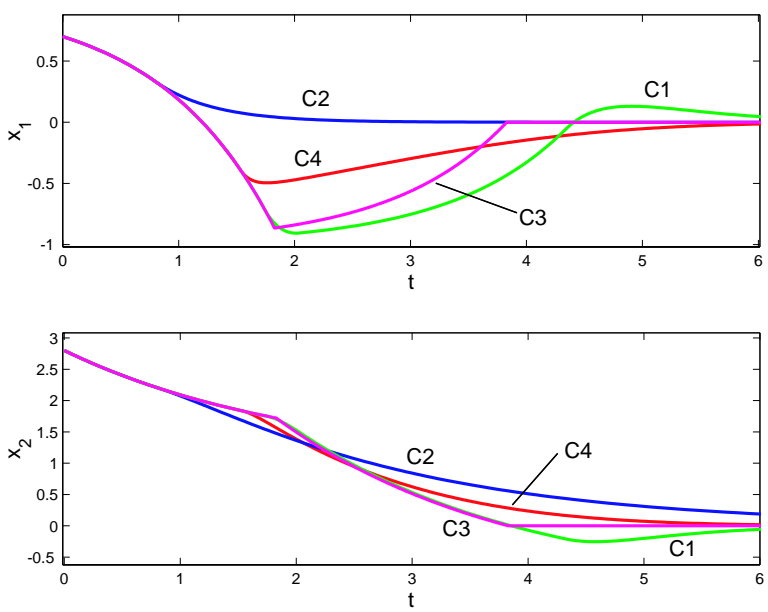

Fig. 4. Time evolution of $x_{1}$ and $x_{2}$.

\section{Chattering in the control signal}

For this example, pseudo-random white noise perturbation is added to the measurements of the state variables. Figure 5 shows the control signal $u$ for all controllers. It is seen that the control signals of $\mathrm{C} 1, \mathrm{C} 2$ and $\mathrm{C} 4$ show no chattering behavior since they are generated by continuous controllers. However, the control signal of C3 shows chattering when the state is near the origin (after $t=3.9[\mathrm{~s}])$. Chattering is also present during the switching from $u=-1$ to $u=1$ at $t=1.826 \ldots 1.833$ [s] (Figure $6)$. 


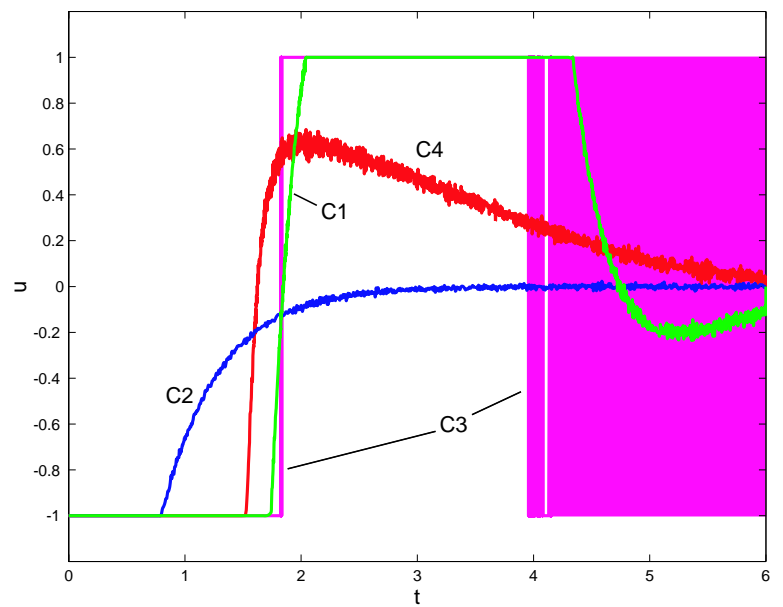

Fig. 5. Time evolution of the input $u$.

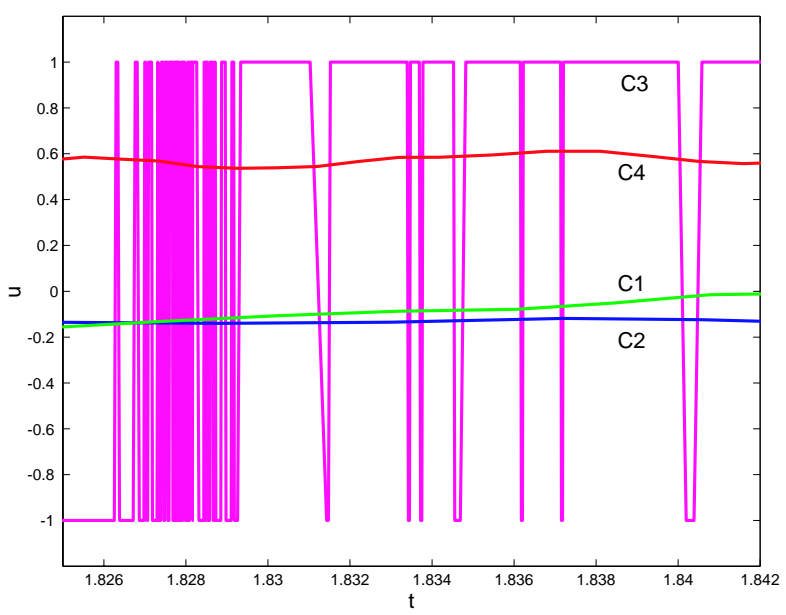

Fig. 6. Time evolution of the input $u$ (zoomed: $t=1.826 \ldots 1.833$ $[\mathrm{s}])$.

\section{CONCLUSION}

In this paper, a simple continuous nonlinear controller for the stabilisation of linear planar systems with one unstable and saturated input was proposed. It was shown that this controller is globally stabilising, i.e. its region of attraction is the null controllable region. Furthermore, it satisfies the desired performance locally around the origin. A third property of this controller is the absence of chattering in the control signal, which is a direct consequence of its continuity.

Though this paper dealt only with planar systems with one stable and one unstable pole, it is hoped that it can be extended to systems with two unstable poles. Also, the case with one unstable pole and multiple stable poles is of interest.

\section{REFERENCES}

[1] J. Alvarez, R. Suárez, and J. Alvarez. Planar linear systems with single saturated feedback. System \& Control Letters, 20:319-326, 1993.
[2] M.S. Branicky. Multiple lyapunov functions and other analysis tools for switched and hybrid systems. IEEE Transactions on Automatic Control, 43(4):475-482, 1998.

[3] J-Y. Favez, B. Srinivasan, Ph. Mullhaupt, and D. Bonvin. Condition for bifurcation of the region of attraction in linear planar systems with saturated linear feedback. CDC 2002, 2002.

[4] T. Hu and Z. Lin. Control Systems with Actuator Saturation: Analyses and Design. Birkhauser, Boston, 2000.

[5] T. Hu, Z. Lin, and L. Qiu. Stabilization of exponentially unstable linear systems with saturating actuators. IEEE Transactions on Automatic Control, 46(6):973-979, 2001.

[6] Z. Lin and A. Saberi. Semi-global exponential stabilization of linear systems subject to input saturation via linear feedback. System \& Control Letters, 21:225-239, 1993.

[7] E.R. Pinch. Optimal Control and the Calculus of Variations. Oxford University Press, New York, 1993.

[8] L. Scibile and B. Kouvaritakis. Stability region for a class of openloop unstable linear systems: Theory and application. Automatica, 36:37-44, 2000.

[9] H.J. Sussmann, E.D. Sontag, and Y. Yang. A general result on the stabilization of linear systems using bounded controls. IEEE Transactions on Automatic Control, 39(12):2411-2424, 1994. 